\title{
The New Era of Consciousness Science - Are We Ready?
}

Can. J. Neurol. Sci. 2010; 37: 713

In this issue Eric Racine and colleagues ${ }^{1}$ provide a comprehensive review of consciousness and its impairments, and provide us with updates on the major ethical controversies. There is no doubt that a new era is at hand. The article by Racine et al could not be more timely.

Chronic disorders of consciousness include coma, vegetative state and the minimally conscious state. The current classification of these disorders of consciousness is clinical and is based on the careful observation and interpretation of clinical signs that support the diagnosis of each syndrome. Despite the advent of new clinical scales ${ }^{2,3}$, the diagnosis, prognosis, support and therapy of chronically unconscious patients remain extremely challenging. It has become evident that diagnosis can be fraught with error, clinical definitions are inexact and prognosis remains difficult.

Indeed, in the last few years, several articles have come out that have forced neurologists to seriously reconsider their basic understanding of vegetative states and coma. There are isolated cases of almost miraculous recovery from a persistent vegetative state with the use of drugs such as Zolpidem ${ }^{4}$ and deep brain stimulation $^{5}$. Behavioural improvement after severe head injury have followed thalamic stimulation or occur spontaneously ${ }^{6,7}$. Such reports have shaken the faith of the medical and general population with regards to the diagnosis and prognosis of this and other serious disorders of consciousness.

Recent technological advances in neuroimaging have produced an impressive array of new techniques for examining both the structure and function of the human brain in vivo (fMRI, PET, tensor imaging, electrophysiological testing). In specialized centers, many of these methods are now being employed in the assessment of patients diagnosed with disorders of consciousness, mapping patterns of residual function and dysfunction and studying the correlation between clinical diagnosis and underlying activity, even when patients cannot behaviourally respond ${ }^{8,9}$. Such advances have the potential to provide important new prognostic indicators, to disentangle differences in outcome on the basis of a greater understanding of the underlying mechanisms responsible and to provide information that will undoubtedly contribute to improved therapeutic choices. But with the advent of these new methods of assessment comes an increasing unease when dealing with the ethical issues associated with the care of the minimally conscious or unconscious patients. A new era of consciousness has begun, but are we ready? We should be prepared to accept that our classification of disorders of consciousness is outmoded. We need to individualize the patient's impairment along a spectrum of brain dysfunction.

At the same time, we should not be too caught up the sensationalism of case reports of "miraculous recoveries" or surprisingly, occasionally preserved aspects of cognitive function in fMRI. Such cases are distinctly uncommon and largely restricted to traumatic brain injury ${ }^{10}$; e.g., they do not happen in global anoxic-ischemic encephalopathy. The ability to generate fMRI responses that require processing of information and producing differential changes in blood flow indicates that many circuits in the brain are still working, but that is not necessarily equivalent to the full spectrum of human conscious experience, which may still be lacking. In addition, such patients usually have severe disability with total dependency.

We live in an interesting and challenging time. What will be the accepted way to make a diagnosis or prognosis? How will we know that either is correct? How do we respect the patient's wishes? Is withdrawal of life support measures an appropriate decision or a form of euthanasia? What about resource allocation?

Issues such as withdrawal of artificial nutrition and hydration, once thought of as settled, are becoming contentious once again under renewed pressure from social groups and religious authorities. Please keep interested, stay tuned and get involved.

Jeanne Teitelbaum, Montreal, Quebec, Canada
G. Bryan Young, London, Ontario, Canada

\section{REFERENCES}

1. Racine E, Rodrigue C, Bernat JL, et al. Observations on the ethical and social aspects of disorders of consciousness. Can J Neurol Sci. 2010;37(6):758-68.

2. Kalmar K, Giacino JT. The JFK coma recovery scale-revised. Neuropsychol Rehabil. 2005;15(3-4):454-60.

3. Whyte J, Katz D, Long D, et al. Predictors of outcome in prolonged posttraumatic disorders of consciousness and assessment of medication effects: a multicenter study. Arch Phys Med Rehabil. 2005;86(3):453-62.

4. Whyte J, Myers R. Incidence of clinically significant responses to zolpidem among patients with disorders of consciousness: a preliminary placebo controlled trial. Am J Phys Med Rehabil. 2009;88:410-18.

5. Shah SA, Baker JL, Ryou JW, Purpura KP, Schiff ND. Modulation of arousal regulation with central thalamic deep brain stimulation. Conf Proc IEEE Eng Med Biol Soc. 2009;2009: 3314-17.

6. Schiff ND, Giacino JT, Kalmar K, et al. Behavioural improvements with thalamic stimulation after severe traumatic brain injury. Nature. 2007;448(7153):600-3.

7. Martin L, Nancarrow D. Colorado woman in vegetative state for 6 years awakens for 3 days. Associated Press. 2007 Mar 08.

8. Monti MM, Vanhaudenhuyse A, Coleman MR, et al. Willful modulation of brain activity in disorders of consciousness. $\mathrm{N}$ Engl J Med. 2010;362:579-89.

9. Owen AM, Coleman MR, Boly M, Davis MH, Laureys S, Pickard JD. Detecting awareness in the vegetative state. Science. 2006; 313:1402.

10. Ropper AH. Cogito ergo sum by MRI. N Eng J Med. 2010;362: 648-9. 